and to hold others accountable for reducing violent death.

\section{The secrets of the National Violent Death Reporting System}

\section{J A Mercy, L Barker, L Frazier}

\section{How the NVDRS provides a foundation for successful violence prevention}

lives public health, as in the rest of our lives, the value and importance of the things we do are not always readily apparent. Sometimes the things we do hold secrets that, once fully revealed, expand our appreciation of their relevance to our goals and aspirations. Such is the case with the National Violent Death Reporting System (NVDRS). On the surface, the NVDRS is a data collection system for violent deaths (that is, homicides and suicides) and in that way is similar to many other surveillance systems that we carry out as part of public health practice. But in reality the NVDRS is much more than it might appear.

SECRET 1: THE NVDRS USES THE POWER OF LINKING DATA

The NVDRS makes better use of data that are already being collected by health, law enforcement, and social service agencies. ${ }^{1}$ The NVDRS, in fact, does not require the collection of any new data. Instead it links together information that, when kept in separate compartments, is much less valuable as a tool to characterize and monitor violent deaths. For example, as part of their death investigation, medical examiners and coroners typically gather information on the presence of alcohol and drugs in the blood and tissues of homicide victims. On the other hand, law enforcement agencies, as part of their criminal investigation, gather details of the circumstances under which the homicide occurred, such as whether the homicide was precipitated by an argument or a gang fight or a robbery. By linking these types of information together, we can now determine the circumstances under which homicide victims are most likely to be intoxicated. Combined with information on the location of homicides, this may shed greater light on the relevance of, for example, alcohol serving policies and guidelines at bars where violence may erupt. It might also help us to anticipate patterns of drug use that could signal the emergence of a homicide epidemic, such as the one that occurred between 1985 and 1993 in conjunction with the distribution of crack or that may be occurring currently with the increase in use and distribution of methaamphetamines. ${ }^{2} 3$ By linking these data, the NVDRS can reveal new insights into the prevention of homicide and suicide and better prepare us for addressing why these violent deaths may be increasing or decreasing.

\section{SECRET 2: THE NVDRS IS A TOOL FOR ACCOUNTABILITY}

The NVDRS will enable federal, state, and local governments and communities to be more accountable for the impact of programs and policies intended to address the problems of homicide and suicide. Both the public and private sectors increasingly set and monitor goals for the many programs and policies that are administered across these sectors. We face the same challenge in violence prevention. This emphasis on accountability requires timely, reliable, and useful data be collected systematically and on an ongoing basis. The NVDRS will facilitate our efforts at accountability in two key ways. First, it provides a tool for goal management that is more timely and useful then other available data systems for violent death. NVDRS data can be used to establish and monitor measurable goals for reductions in homicide and suicide (for example, a goal to reduce the homicide rate among adolescents). Second, as the NVDRS is implemented in more and more states (it is currently in 17 states) and data are accumulated over time, it will become increasingly useful for directly evaluating the impact of state and local violence prevention policies and programs. For example, NVDRS data could be used in conjunction with other information to determine if temporal and geographic variations in state policies addressing welfare reform are associated with differences in rates of intimate partner homicide. The NVDRS thus provides information which enables anyone working in the field of violence prevention to be accountable

\section{SECRET 3: THE NVDRS FOSTERS COLLABORATIONS FOR PREVENTION}

The NVDRS fosters cooperation and collaboration among federal, state, and local agencies and organizations that must work together in order to successfully prevent violence. If we are to prevent violence, the cooperation and collaboration of a broad range of agencies and organizations will be required. ${ }^{4}$ State and local health departments, police departments, the media, social services, and vital statistics agencies are just a few of the entities that need to work together to develop, implement, and broadly disseminate effective prevention strategies. Many of these organizations have not worked together in the past or have worked together on a limited basis. These organizations all play a role in at least one phase of collecting and disseminating data for the NVDRS. For example, state health departments coordinate NVDRS data collection and can use NVDRS data to identify priorities for public health action and to identify appropriate prevention strategies for the communities they serve. Police departments collect data that are essential for characterizing the circumstances under which homicides occur (for example, the percentage of homicides precipitated by drug related crimes). However, police departments also play an important role in enforcing laws that might deter some forms of violence. The media can use NVDRS data as background for news stories about homicide or suicide, but might also disseminate key violence prevention messages. Child protection service agencies provide important information on child abuse related homicides to child death review teams that inform the NVDRS and also provide services to high risk children and their families that may be important in preventing homicide. State vital statistics agencies provide information from death certificates that is essential for the identification of violent deaths for the NVDRS and can also use NVDRS data to detect inconsistencies in their policies for assigning manners of deaths in death certificates. ${ }^{5}$ In the process of collecting and linking together NVDRS data, relationships and collaborations are established among key organizations as part of that process that can and do carry over into violence prevention activities. The NVDRS serves as a locus around which collaborative relationships that contribute to violence prevention are established. 


\section{SECRET 4: THE NVDRS EMPOWERS PEOPLE WITH INFORMATION}

NVDRS data will facilitate the involvement of the public in violence prevention programs and activities because everyone will eventually have access to and be able to use its data. As a result, the public will gain better insight into the circumstances surrounding violent deaths and this knowledge will help them more clearly understand roles that they can play in prevention. NVDRS data are currently only available to Centers for Disease Control and Prevention and state public health researchers. Plans are underway to produce a public use data set, with some restrictions to protect confidentiality and privacy. In addition, the first steps are being taken to make the NVDRS even more accessible through a web-based system that allows anyone with access to the internet to ask basic questions of the data and get answers almost instantaneously. This system will be modeled on the Web-based Injury Statistics Query and Reporting System (WISQARS), a system currently in place that allows one to get basic data on injury related deaths. ${ }^{6}$ So, for example, in the not too distant future, if a citizen of Wisconsin wants to know how many suicides occurred in their state in 2004 among high school aged youth by means of drug overdose and how this number has changed in recent years, he or she will be able get that information in a matter of minutes. This kind of access to NVDRS data will empower people with facts they can use to become more informed about violent death and more directly involved in efforts to prevent it.

These are the secrets of the NVDRS. The NVDRS, however, will continue to reveal secrets about the nature and prevention of violent deaths as the system spreads to all states and data are accumulated. But as you can see the NVDRS is much more then just another data collection system. It provides a foundation upon which many activities and processes necessary for successful violence prevention can be built. Clearly if we are ultimately going to be successful in preventing the various forms of violence that contribute to homicide and suicide we will need much more than the NVDRS but, just as clearly, the NVDRS is a very critical piece of the puzzle. Let's not keep the NVDRS a secret any longer.

Injury Prevention 2006;12(Suppl II):ii1-ii2. doi: 10.1136/ip.2006.012542

\section{Authors' affiliations}

J A Mercy, L Barker, L Frazier, Centers for Disease Control, National Center for Injury Prevention and Control, Atlanta, GA, USA
Correspondence to: Dr J A Mercy, Centers for Disease Control and Prevention, National Center for Injury Prevention and Control, 4770 Buford Highway, NE, Mailstop K-68, Atlanta, GA 30341, USA; jam2@cdc.gov

Accepted 3 August 2006

The findings and conclusions in this report are those of the authors and do not necessarily represent the views of the Centers for Disease Control and Prevention.

\section{REFERENCES}

1 Azrael D, Barber C, Mercy J. Linking data to save lives: recent progress in establishing a National Death Reporting System. Harvard Health Policy Review $2001 ; 2: 38-42$

2 Blumstein A. Youth violence, guns and the illicitdrug industry. J Crim Law Criminol 1995;86:10-36

3 Johnson B, Golub A, Dunlap E. The rise and decline of hard drugs, drug markets, and violence in inner-city New York. In: Blumstein A, Wall-man J (eds). The crime drop in America. New York: Cambridge University Press, 2000:164-206.

4 Mercy JA, Rosenberg ML, Powell KE, et al. Public health policy for preventing violence. Health Aff 1993; 12:7-29.

5 Breiding MA, Wiersema B. Variability of undetermined manner of death classification in the US. Inj Prev 2006;12(Suppl II):ii49-ii54.

6 Centers for Disease Control and Prevention. WISQARS Injury Mortality Reports, 1981-2003. Atlanta, GA: National Center for Injury Prevention and Control, Centers for Disease Control and Prevention; 2006, Available at http://www.cdc.gov/ncipc/wisqars (accessed September 2006). 